\title{
UPPER AND LOWER COMPLEMENTATION IN A MODULAR LATTICE
}

\author{
S. P. AVANN
}

In this paper we define upper and lower complements of $a \in L$, always a finite modular lattice, such that these become an ordinary complement of $a$ when $L$ is complemented (Theorem 8). Uniqueness of upper or of lower complements for all $a \in L$ implies $L$ is distributive (Theorem 5). Our principal result is a set of 8 equivalent conditions for uniqueness of upper or lower complement of a particular element $a \in L$ (Theorem 4).

We shall employ $\supset, \supseteq,>$ for proper inclusion, inclusion, and covering respectively, and $C, \subseteq,<$ for their duals. Unit and zero of $L$ will be $u$ and $z$ respectively. Otherwise, notation and terminology of Birkhoff's Lattice theory [2] will be adhered to.

We shall denote by $A$ the set $A=\{x \in P \mid x \subseteq a\}$ where $P$ is the set of join-irreducibles of $L$ partially ordered by the ordering relation of $L$. Elementary properties enjoyed by these sets $A$ are: (1) $a=\bigcup_{x \in A} x$; (2) $A=B$ if and only if $a=b$; (3) $A \supset B$ if and only if $a \supset b$; (4) $c=a \cup b$ and $d=a \cap b$ imply $C \supseteq A+B$ and $D=A \cdot B$ where $(+)$ and $(\cdot)$ are point-set sum and product.

We denote by $a^{*}$ the join of all elements covering $a$ and by $a_{*}$ the meet of all elements covered by $a$. We define $u^{*}=u$ and $z_{*}=z$.

The quotient $c / a$ is an upper transpose of $b / d$ and $b / d$ is a lower transpose of $c / a$ if and only if $c=a \cup b$ and $d=a \cap b$.

In Lemma 1, Theorems 5 and 8, and Corollary to Theorem 9 use of parenthesized words yields the dual theorem.

LEMMA 1. In a modular lattice, $c / a(b / d)$ is a maximal (minimal) complemented quotient in the complete set $Q$ of projective quotients to which it belongs if and only if $c=a^{*}\left(d=b_{*}\right)$.

Proof. First suppose $c / a$ is a maximal complemented quotient and consider $a_{i}>a$. Then $a_{i} \subseteq c$; otherwise $a_{i} \cap c=a$ and $c \cup a_{i} / a_{i}$ is a proper upper transpose of $c / a$ contradicting the maximality of $c / a$. Applying Theorem $6[2, \mathrm{p} .105]$ we obtain $c=\mathrm{U}_{a_{i}>a} a_{i}=a^{*}$. Conversely, if $c=a^{*}$, then $c / a$ is complemented by the same Theorem 6 . Assume there exists a proper upper transpose $e / f$ of $c / a$. Then for some $a_{1}, f \supseteq a_{1}>a=f \cap c$. But $a_{1} \subseteq a^{*}=c$ by definition, which leads to the contradiction $a_{1} \subseteq f \cap c=a$. Hence $c / a$ has no proper upper transpose.

Received by the editors March 16, 1959. 
Definition 1. In a modular lattice the numerator $a_{U}^{\prime}$ of a minimal quotient projective with $a^{*} / a$ is called an upper complement of $a$. It is called a direct upper complement if the minimal quotient is a lower transpose of $a^{*} / a$, otherwise $a_{U}^{\prime}$ is called indirect. Dually, the denominator $a_{L}^{\prime}$ of a maximal quotient projective with $a / a_{*}$, is called a lower complement of $a$. It is called direct if the maximal quotient is an upper transpose of $a / a_{*}$, otherwise indirect. An upper associate $a_{U}$ of $a$ is the denominator of a maximal quotient projective with $a^{*} / a$, and a lower associate $a_{L}$ of $a$ is the numerator of a minimal quotient projective with $a / a_{*}$.

In view of Lemma $1 a$ is both an upper and a lower associate of itself.

THEOREM 1. In a modular lattice $b$ is a (direct) upper complement $a_{U}^{\prime}$ of $a$ if and only if $a$ is a (direct) lower complement $b_{L}^{\prime}$ of $b$.

Proof. Suppose $b=a_{U}^{\prime}$ is determined by the minimal quotient $b / d$ projective to $a^{*} / a$, the latter being complemented by Lemma 1 . Projective quotients are isomorphic, so that $b / d$ is also complemented as well as minimal. Hence $d=b_{*}$ by Lemma 1 . Again by Lemma 1 $a^{*} / a$ is maximal and is projective to $b / b_{*}$. Hence $a=b_{L}^{\prime}$. The converse follows by duality. The relationship between $a$ and $b$ is direct if and only if $a^{*} / a$ and $b / b_{*}$ are upper and lower transposes of one another respectively.

THEOREM 2. In a modular lattice $L$ there exists at least one direct upper complement and one direct lower complement of each $a \in L$.

Proof. The theorem follows by the transitivity of lower and upper transposition. If $a^{*} / a$ has no proper lower transpose, $a^{*}$ is the unique upper complement of $a$, and dually.

THEOREM 3. In a modular lattice $L$ the number of upper (lower) complements of each $a \in L$ is equal to the number of upper (lower) associates of $a$.

Proof. The theorem follows directly from Definition 1 and Theorem 6.2 of [1], which asserts that in a complete set $Q$ of projective complemented quotients, the number of maximal quotients is equal to the number of minimal quotients.

CoRollary. For $b=a_{U}^{\prime}$ and $a=b_{L}^{\prime}$ in a modular lattice, the number of upper complements of $a$ is equal to the number of lower complements of $b$.

THEOREM 4. In a modular lattice $L$ let $b$ and $a$ be respectively $a$ direct 
upper complement and a direct lower complement of the other and let $Q$ be the complete set of projective complemented quotients to which $a^{*} / a$ and $b / b_{*}$ belong. The following conditions are equivalent.

(A) $b$ is the unique upper complement of $a$.

(B) $a$ is the unique lower complement of $b$.

(C) Every $e / f \in Q$ is a lower transpose of $a^{*} / a$ and an upper transpose of $b / b_{*}$.

(D) $a^{*} / b_{*}$ is (isomorphic to) the direct product $a / b_{*} \times b / b_{*}$.

(E) $a$ and $b$ are $a$ distributive pair: all 6 distributive laws hold for $a, b$ and every $g \in L$.

(F) $A^{*}=A+B$.

(G) $A^{*}-A=B-B_{*}$.

(H) $E-F$ is an invariant subset of $P$, the set of all join-irreducible elements of $L$, for all $e / f \in Q$.

Proof. (A) implies (B). Referring to Theorem 1 and its proof, when $b$ is the only upper complement of $a, b / b_{*}$ is the unique minimal quotient of $Q$. From [1, Theorem 6.2] quoted in the proof of Theorem 3 we conclude that there exists exactly one maximal quotient of $Q$, which must be $a^{*} / a$ by Lemma 1 . Hence $a$ is the unique lower complement of $b$.

(B) implies (A) by duality.

(A) and (B) imply (C). Suppose there exists in $Q$ a quotient $e / f$ which is not a lower transpose of $a^{*} / a$. By Lemma 1 a maximal upper transpose of $e / f$ is of the form $g^{*} / g$ and is maximal in $Q$. Since lower transposition is transitive, $g^{*} / g$ must also fail to be a lower transpose of $a^{*} / a$. Thus $g$ is a lower complement of $b$ and is distinct from $a$, contradicting hypothesis (B). Thus every $e / f \in Q$ is a lower transpose of $a^{*} / a$ and by a dual argument is an upper transpose of $b / b_{*}$.

(C) implies (A) and (B) since $a^{*} / a$ and $b / b_{*}$ are obviously the unique maximal and the unique minimal quotients respectively of $Q$.

(C) implies (D). As an immediate corollary of Theorem $7[2$, p. 73] and its dual, (D) is valid if and only if for every $k \in a^{*} / b_{*}$ $k=(k \cap a) \cup(k \cap b)=(k \cup a) \cap(k \cup b)$. Assume (D) is false and that $h$ is minimal in $a^{*} / b_{*}$ such that $h \supset(h \cap a) \cup(h \cap b)$. Obviously $h \supset b_{*}$. Let $g$ be an arbitrary element of $a^{*} / b_{*}$ covered by $h$. Then $h \supset(h \cap a) \cup(h \cap b) \supseteq(g \cap a) \cup(g \cap b)=g$. It follows that $g=(h \cap a) \cup(h \cap b)$ and that $g$ is unique; i.e. $h$ is a join-irreducible of $a^{*} / b_{*}$ ( $h$ covers only one element $g$ ). Moreover, in $a^{*} / b_{*} g, b \supseteq h \cap b$ $\supseteq g \cap b$ from which $h \cap b=g \cap b$. Next, $h>g=g \cup(b \cap g)=g \cup(b \cap h)$ $=(g \cup b) \cap h$ by the modular axiom. Therefore by the upper semimodularity axiom $h \cup b=(g \cup b) \cup h>g \cup b$. We observe for later reference that $h \cup b / h$ is an upper transpose of $g \cup b / g$. Now $t \leftrightarrow t \cap a$ deter- 
mines Dedekind's natural isomorphism [2, Theorem 6, p. 73] between $a^{*} / b$ and its lower transpose $a / b_{*}$. Hence $(h \cup b) \cap a>(g \cup b) \cap a$ in $a / b_{*}$. Validity of one distributive law $g \cap(a \cup b)=g \cap a^{*}=g$ $=(g \cap a) \cup(g \cap b)$ implies validity of all 6. Hence $(h \cup b) \cap a>(g \cup b) \cap a$ $=(g \cap a) \cup(b \cap a)=(g \cap a) \cup b_{*}=g \cap a$. Again, $(g \cap a) \cup b$ $=(g \cup b) \cap(a \cup b)=(g \cup b) \cap a^{*}=g \cup b$, together with $(g \cap a) \cap b=g \cap b_{*}=b_{*}$, show that $g \cup b / g \cap a$ is an upper transpose of $b / b_{*} \in Q$ and therefore also complemented. Hence by $[2$, p. 105 , Theorem 6, L7 $\left.7^{\prime}\right] g \cup b$ is the join of elements covering $g \cap a$. Applying the modular axiom, $h \cup b=a^{*} \cap(h \cup b)=[(g \cup b) \cup a] \cap(h \cup b)$ $=(g \cup b) \cup[a \cap(h \cup b)]$ likewise is a join of elements covering $g \cap a$. Thus $h \cup b / g \cap a$ satisfies $\mathrm{L}^{\prime}$ and is complemented. Since $h$ is a joinirreducible of $a^{*} / b_{*}$, it is a join-irreducible of the complemented quotient sublattice $h \cup b / g \cap a$ and must cover $g \cap a$. Thus $h>g=g \cap a$. We have now shown $h \cup b / h$ is an upper transpose of $g \cup b / g=g \cup b / g \cap a$, which in turn is an upper transpose of $b / b_{*}$. Hence by transitivity of upper transposition and hypothesis (C) $a^{*} / a$ is an upper transpose of $h \cup b / h$ so that $h \subseteq a$. Thus the initial assumption that (D) is false leads to the contradiction $g=(h \cap a) \cup(h \cap b)=h \cup(h \cap b)=h$.

(D) implies (C). Let $e / f$ be arbitrary in $Q$. By the extended semimodularity axiom $[2$, p. 100, (2) $]$ and its dual there exists between $a^{*} / a$ and $e / f$ a sequence of upper and lower transposes, each a "covering" transpose: $a^{*} / a=e_{0} / f_{0} \sim e_{1} / f_{1} \sim \cdots \sim e_{n} / f_{n}=e / f$ where $e_{i-1}>e_{i}$ and $f_{i-1}>f_{i}$ or $e_{i-1}<e_{i}$ and $f_{i-1}<f_{i}(i=1,2, \cdots, n)$. We shall prove by an induction on $i$ that all these quotients satisfy (C). Trivially for $i=0,(\mathrm{C})$ is satisfied. Assume (C) is satisfied for $i$. Suppose $e_{i+1} / f_{i+1}$ is an upper covering transpose of $e_{i} / f_{i}$, hence is also an upper transpose of $b / b_{*}$. Assume $a \supseteq f_{i+1}$. Then $f_{i+1} \supset a \cap f_{i+1} \supseteq f_{i}$ demands $f_{i+1}>a \cap f_{i+1}=f_{i}$. By the upper semi-modularity axiom $a \cup f_{i+1}>a$. But then $a \cup f_{i+1} \subseteq a_{1}^{*}$. Then $f_{i+1}=a^{*} / b_{*}=a / b_{*} \times b / b_{*}$ requires $f_{i+1}$ $=\left(a \cup f_{i+1}\right) \cap\left(b \cup f_{i+1}\right)=\left(a \cup f_{i+1}\right) \cap e_{i+1}$. But $\left(a \cup f_{i+1}\right) \cup e_{i+1}=\left(a \cup f_{i+1}\right)$ $\cup\left(b \cup f_{i+1}\right)=a^{*} \cup f_{i+1}=a^{*}$. We have verified that $a^{*} / a \cup f_{i+1}$ is an upper transpose of $e_{i+1} / f_{i+1}$. Hence $a^{*} / a$ is projective with and therefore isomorphic to a proper sublattice $a^{*} / a \cup f_{i+1}$ of itself, a contradiction. Thus $a \supseteq f_{i+1}$. We now obtain $e_{i+1} \cup a=\left(f_{i+1} \cup b\right) \cup a=a \cup b=a^{*}$ and by the modular law $e_{i+1} \cap a=\left(f_{i+1} \cup b\right) \cap a=f_{i+1} \cup(b \cap a)=f_{i+1} \cup b_{*}$ $=f_{i+1}$. This verifies that $e_{i+1} / f_{i+1}$ is a lower transpose of $a^{*} / a$ as well as an upper transpose of $b / b_{*}$, which is condition (C). If $e_{i+1} / f_{i+1}$ were rather a lower covering transpose of $e_{i} / f_{i}$, (C) follows by a dual argument. The basis of the induction is now complete, and $e / f$ satisfies (C).

(D) implies (F). Let $x$ be an arbitrary join-irreducible in $A^{*}: x \subseteq a^{*}$. 
Let $g$ be a minimal element of $a^{*} / b_{*}$ such that $x \subseteq g$. By hypothesis $a^{*} \supseteq g=(g \cup a) \cap(g \cup b) \supseteq(x \cup a) \cap(x \cup b) \supseteq x \cup(a \cap b)=x \cup b_{*} \supseteq x, \quad b_{*}$. By minimality $g=(x \cup a) \cap(x \cup b)=x \cup(a \cap b)$. Hence $x, a, b$ form a distributive set and $(x \cap a) \cup(x \cap b)=x \cap(a \cup b)=x \cap a^{*}=x$. Joinirreducibility demands $x \cap a=x, x \subseteq a, x \in A$ or $x \cap b=x, x \subseteq b, x \in B$. Hence $x \in(A+B)$, so that $A^{*} \subseteq A+B \subseteq A^{*}$ yields equality.

(F) implies (E). Let $g$ be arbitrary in $L$ and let $r=(a \cup b) \cap g$, $s=a \cap g, t=b \cap g, v=(a \cap g) \cup(b \cap g)=s \cup t$. Then $R=A^{*} \cdot G$ $=(A+B) \cdot G=A \cdot G+B \cdot G=S+T \subseteq V \subseteq R$, since $v \subseteq r$. Hence $R=V$ and $r=v$.

(E) implies (D). For arbitrary $g \in a^{*} / b_{*} g=g \cup b_{*}=g \cup(a \cap b)$ $=(g \cup a) \cap(g \cup b)$, which is the necessary and sufficient condition, cited earlier, for the desired direct product condition.

(F) implies (G). $A^{*}=A+B$ implies $A^{*}-A=B-A \cdot B=B-B_{*}$.

(G) implies (F). $A^{*}=A+\left(B-B_{*}\right)=A+(B-A \cdot B)=A+B$.

(G) implies $(\mathrm{H})$. We already have shown the equivalence of (A)-(G) inclusive. By (C) and (F) we obtain $E=E \cdot A^{*}=E \cdot(A+B)$ $=E \cdot A+E \cdot B=F+B$. Therefore $E-F=B-B \cdot F=B-B_{*}=A^{*}-A$ for all $e / f \in Q$.

(H) implies (G) trivially. This completes the proof of Theorem 4.

TheOREM 5. A modular lattice $L$ is distributive if and only if each $a \in L$ has exactly one upper (lower) complement.

Proof. First, suppose each $a \in L$ has exactly one upper complement. Assume that $L$ is nondistributive. It will then have a nondistributive modular sublattice of order 5 with coverings: $c>e_{1}, e_{2}, e_{3}$ $>d$ for distinct $e_{1}, e_{2}, e_{3}$. Let $c \cup a / a$ be a maximal upper transpose of the prime quotient $c / e_{1}$ and therefore a maximal prime quotient of the complete set $Q$ of projective prime (trivially complemented) quotients to which the prime quotients of $c / d$ belong. By Lemma 1 $c \cup a=a^{*}>a$. Let $b / b \cap d$ be a minimal lower transpose of $e_{2} / d$ hence a minimal lower transpose of $a \cup c / a$. Thus $b=a_{U}^{\prime}$, the unique upper complement of $a$, and $b / b \cap d$ is $b / a \cap b$. Likewise a minimal lower transpose of $e_{3} / d$ must be $b / a \cap b$. But then $e_{2}=b \cup d=e_{3}$, a contradiction. Hence $L$ is distributive.

Conversely, suppose $L$ is distributive. Consider arbitrary $a \in L$ with $b=a_{U}^{\prime}, a=b_{L}^{\prime}$. Condition (E) of Theorem 4 holds, hence also conditions (A) and (B) by that Theorem.

Definition 2. The quotient $a / b$ of a modular lattice $L$ is called a central sublattice of $L$ if and only if $a / b$ is complemented and has no upper and no lower transposes. 
THEOREM 6. In a modular lattice $L a / b$ is a central sublattice if and only if $a=b^{*}$ and $b=a^{*}$.

Proof. This follows directly from Definition 2 and Lemma 1.

THEOREM 7. In a modular lattice $L$ if $b$ is both an upper and a lower complement of a then $a^{*}=b^{*}, a_{*}=b_{*}$, and $a^{*} / b_{*}$ is a central sublattice.

Proof. By Theorem $1 a$ is also both an upper and a lower complement of $b$. Hence $a^{*}=a \cup b=b^{*}$ and $b_{*}=a \cap b=a_{*}$. Both $b / b_{*}$ and $a / a_{*}$ are complemented. By [2, p. 105, Theorem 6, L7'] $a$ and $b$ are both joins of elements covering $a_{*}=b_{*}$, therefore, so is $a^{*}=b^{*}$, and by the same Theorem $a^{*} / a_{*}$ is complemented. If $a^{*} / a_{*}$ has an upper transpose, so would also the subquotient $a^{*} / a$ by Dedekind's Transposition Principle, $[2$, Theorem 6, p. 73] violating the maximality asserted by Lemma 1 . Thus $a^{*} / a_{*}$ has no upper transpose, and by a dual argument has no lower transpose. Hence $a^{*} / a_{*}$ is central.

THEOREM 8. For an arbitrary element a of a complemented modular lattice $L, b$ is an ordinary complement of $a$ if and only if $b$ is an upper (lower) complement of $a$.

Proof. All quotients of $L$ are complemented. Hence $a^{*}=u=b^{*}$ and $a_{*}=z=b_{*}$. The equivalence then follows directly from application of the definitions of each of the types of complements.

Theorem 9 and its corollary are decidedly stronger than the converse of Theorem 8 .

TheOREM 9. If there exists one element a of a modular lattice $L$ for which $b \in L$ is simultaneously an upper, a lower, and an ordinary complement of $a$, then $L$ is complemented.

Proof. By Theorem $7 a^{*}=a \cup b=u, a_{*}=a \cap b=z$, and $a^{*} / a_{*}=u / z$ $=L$ is complemented.

CoRollary. If in a modular lattice $a_{*}=z\left(a^{*}=u\right)$ and $b$ is both an ordinary and an upper (a lower) complement of a, then $L$ is complemented.

We note that $a_{*}=z$ whenever $a=z$ or $a>z$.

\section{BIBLIOGRAPHY}

1. S. P. Avann, Dual symmetry of projective sets in a finite modular lattice, Trans. Amer. Math. Soc. vol. 89 (1958) pp. 541-558.

2. G. Birkhoff, Lattice theory, rev. ed., Amer. Math. Soc. Colloquium Publications, vol. $25,1948$.

UNIVERSITY OF WASHINGTON 